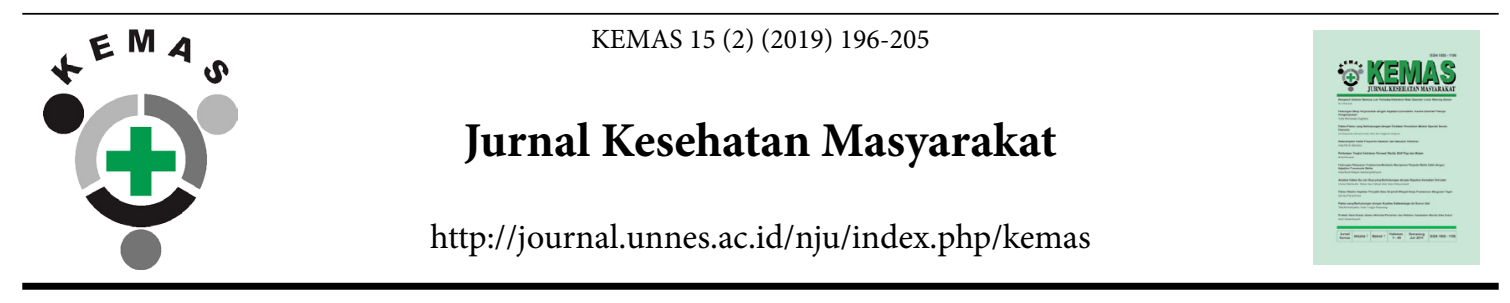

\title{
Current Evidence on the Effect of Beetroot Juice During Exercise Efforts
}

\author{
Sun $\mathrm{He}^{\bowtie}$, Tengku Fadilah Tengku-Kamalden \\ Department of Sport Studies, Universiti Putra Malaysia, Kuala Lumpur, Malaysia
}

\begin{tabular}{l} 
Article Info \\
\hline Article History: \\
Submitted July 2019 \\
Accepted October 2019 \\
Published November 2019 \\
\hline Keywords: \\
beetroot, beetroot juice, \\
supplementation, \\
exercise effort \\
\hline DOI \\
https://doi.org/10.15294/ \\
kemas.v15i2.14349
\end{tabular}

\begin{abstract}
Some athletes consume supplements to improve the effect of training and achieve optimal performance during competition. Among the different supplements, beetroot juice has been indicative of improved nitric oxide (NO) concentration in blood, which can promote vasodilation, blood flow, gene expression, mitochondrial efficiency and gas exchange. Different methods have been proposed to classify beetroot juice as an egorgenic aid that can improve the performance of athletes. The objective of this paper is to review methods chosen to gain greater and detailed understanding regarding current issues about the effect of beetroot juice as an ergogenic aid during exercise efforts. Based on Pubmed database, conceptual keywords were input (beet or beetroot, nitrate or nitrite, supplement or supplementation, and exercise, efforts, and physical exercise), and covered literature from 2010 to 2019. Animal researches, review and meta-analyses articles, and inaccessible full-text, and studies that did not use beetroot juice supplementation were excluded, leaving 170 researches available. CiteSpace (version V) showed that the United States and several European countries occupied a dominant position in the carriers of beetroot juice research network. Current prominent research areas were effects of dietary NO3- supplementation, including blood pressure, $\mathrm{O} 2$ cost of submaximal exercise, and exercise performance. The optimal strategy for dietary NO3- supplementation and potential effects of dietary NO3- supplementation on intermittent exercise performance involve muscle fiber type recruited and the Yo-Yo intermittent recovery test..
\end{abstract}

\section{Introduction}

Nitric oxide (NO), the colorless, odorless gas is insoluble in water, was first discovered by a great scientist named Joseph Priestly. However in the beginning the NO was considered as the toxic gas, because it can contribute the depletion of ozone layer. Even, when it reacts with oxygen, it forms a corrosive gas, nitrogen dioxide $\left(\mathrm{NO}_{2}\right)$, which reacts with water to produce nitric acid. The equation is $3 \mathrm{NO}_{2}+$ $\mathrm{H}_{2} \mathrm{O}=2 \mathrm{HNO}_{3}+\mathrm{NO}$. In 1980' s, not only it can be synthesized in the human body from $\mathrm{L}$-arginine (a kind of amino acid), but it was recognized as the endothelium derived relaxing factor (EDRF), which had an effect on diastolic blood vessels and blood flow. Until 1998 many researchers launched on exploring the role of
NO in the processes of physiology. Further, it was also evident that in our diet, nitrate $\left(\mathrm{NO}_{3}^{-}\right)$and nitrite $\left(\mathrm{NO}_{2}^{-}\right)$, which belonged to anions were previously recognized as toxic substance, were endogenously produced as oxidation products of NO. And NO pathway was discovered $\left(\mathrm{NO}_{3}^{-}-\mathrm{NO}_{2}^{-}-\mathrm{NO}\right)$, meaning that there was the sequential reduction from $\mathrm{NO}_{3}^{-}-\mathrm{NO}_{2}^{-}-\mathrm{NO}$ pathway (Todd, Grabowski, Helfrich, Benjamin, \& Ralston, 1994), and some other nitrogen oxides produced it the same time. Therefore, this pathway as another system to ensure NO generation when L-arginine - NO pathway inefficiency (Fig. 1). Skeletal Muscle may suffer conditions of low $\mathrm{O} 2$ tension and PH during contraction (Richardson, Leigh, \& Wagner, 1995) which means that this pathway 
may be important during exercise efforts.

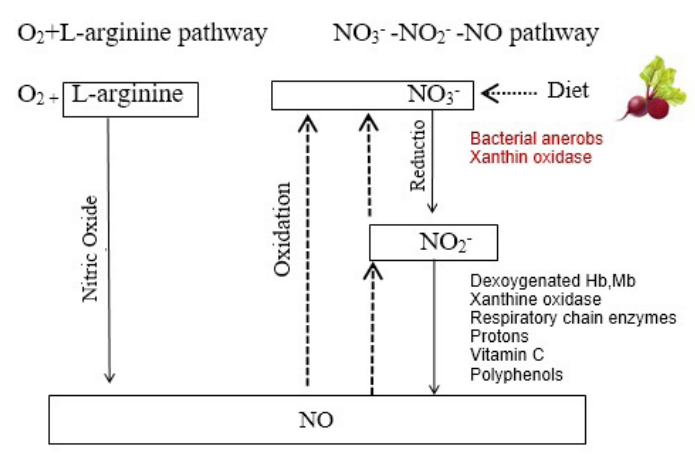

Fig. 1 Two parallel pathways for human body synthesizes the nitric oxide (NO). Left: L-arginine with $\mathrm{O} 2$ pathway. Right: nitrate (NO3-) -nitrite(NO2-)-NO pathway. $\mathrm{Hb}$ (haemoglobin), $\mathrm{Mb}$ (myoglobin) (source from Jones et al. 2014)

Meanwhile, some literature highlight that in the human mouth $25 \%$ of nitrate can reduce to $\mathrm{NO}_{2}$ - by $\mathrm{NO}_{3}^{-}$reductase (Lundberg \& Govoni, 2004). A part of $\mathrm{NO}_{2}$-reduce to $\mathrm{NO}$ through the action of stomach acids, which is absorbed in the gut eventually (Raat, Shiv \& Gladwin). And some of the $\mathrm{NO}_{2}$ enter in the bloodstream, with the condition of low oxygen, can convert into NO (Lundberg \& Govoni, 2004)(Figure 2).

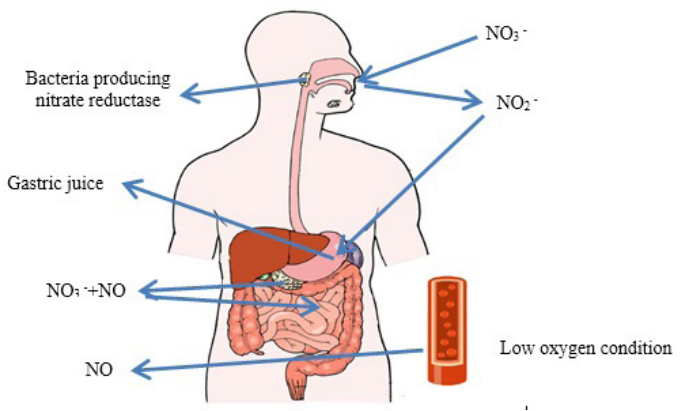

Fig. 2 The process of NO3- in beetroot juice convert into NO.

Various studies have assessed the efficacy of beetroot juice as the supplementation can benefit the perfomance during different types of exercise (Kelly, Vanhatalo, Wilkerson, Wylie, \& Jones, 2013). Two articles have explored this kind of supplementation used in endurance exercise, which actions in the oxidative metabolism, may be make NO becomes the potential factor limits the performance (Domínguez et al., 2018). Therefore, several studies have shown that the beetroot juice as the $\mathrm{NO}_{3}^{-}$supplementation can lower $\mathrm{VO}_{2 \max }$ by $-6 \%$ during a swimming test (Pinna et al., 2014)], and -3\% during a kayaking test at $60 \% \mathrm{VO}_{2 \max }$ (Muggeridge, Howe, Spendiff, Pedlar, James, Easton, et al., 2013); improve performance by $12-17 \%$ during cycle ergometry tests at $60-90 \% \quad \mathrm{VO}_{2 \max }$ by recreation athletes (Thompson, K. et al., 2014) shorten time by $2.8 \%$ and $2.7 \%$, conducted by cyclists in $4 \mathrm{~km}$ and $16 \mathrm{~km}$ respectively (Larsen, Weitzberg, Lundberg, \& Ekblom, 2007). On the other hand, increasing number of studies show that the beetroot juice as an ergogenic aid can benefit fast twitch muscle fibers during intermittent high-intensity exercise (Thompson, Wylie, Fulford, \& Kelly, 2015). Several studies found that beetroot juice as the supplementation can effect at: increasing $+4 \%$ average power during a Kayak ergometer $5 \times 10$ s sprint-test with trained kayakers (Muggeridge, Howe, Spendiff, Pedlar, James, Easton, et al., 2013); a work volume improved by $5 \%$ at the half-time simulation match in the $2 \times[5 \times(6 \mathrm{~s}$ cycle ergometry sprint $+14 \mathrm{~s}$ active recover )] with recreation team sport players(male, $\mathrm{n}=16$ ) (Thompson, Wylie, et al., 2015); rising improvements to $20 \%$ conducted by cycle ergometer test at $170 \% \mathrm{VO}_{2 \text { max }}$, followed by 30s recovery (Aucouturier, Boissiere, Pawlakchaouch, Cuvelier, \& Gamelin, 2015). Over the past decade, most research in beetroot juice supplementation has emphasized that it can be an ergogenic aid to improve performance in exercise efforts. However, visualization analysis of dominant research area is unavailable. This study based on Pubmed database and describe the Citespace (version V) application , the retrieval strategy for data collection, the parameter design for scientific analysis, and analyzed the result in visualization map to show the research status of beetroot juice in exercise efforts. From the map of country, institution, time zone and authors cooperation network evaluate dominant research areas and provide clear evidence for future researchers.

\section{Methods}

Data Search Strategy and Sample Information

In this study, the data are from the Pubmed, which provides medical papers search. 
How to set up the retrieval expression to make the result include all the research results of beetroot juice have assessed the effect in exercise effort is the first problem to be considered. Three conceptual key words are adopted: beet OR beetroot OR beetroot juice, nitrate OR nitrite, supplement or supplementation, AND exercise, efforts, AND physical exercise. The time rang is 2010-2019 (the data of retrieval and download is 25th April 2019), and limited factors "Clinical Trial", "Full Text", "Humans" 170 literature were retrieved (showed by Table $1)$.

Tabel 1. Literature Retrieval Strategy

\begin{tabular}{ll}
\hline Step & Content \\
\hline Data resource & beet OR beetroot OR \\
& nitrate OR nitrite \\
& (concept) AND \\
supplement OR & nutrition OR "sport \\
& nutrition" (concept2) \\
& AND exercise OR \\
& sport OR "physical \\
& activity" OR effort \\
& OR athlete (concept3) \\
& $2010-2019$ \\
Time Span (year) & English \\
Literature Language & Full Article \\
Literature Types & 170 \\
Search Results (articles) &
\end{tabular}

Sample Information and Reliability

From chart 1, we can see the increasing number of articles, and the literature after 2016 accounts for $54 \%$, which can objectively reflect the lastest research.

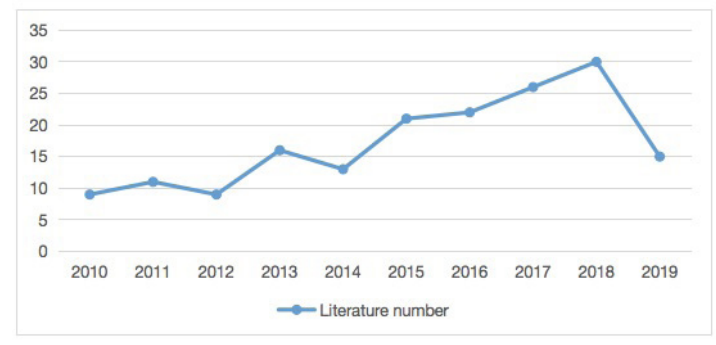

Chart 1. Increasing number of articles, and the literature

Indicator Selection

Centrality (C): an index to measure the importance of nodes in the network. CiteSpace uses this indicator to discover and measure the importance of documents, and uses purple rings to highlight such documents (or authors, journals, institutions, etc.) (Chen, C., 2017). Software Introduction and Research Process

This study is based on the JAVA platform of CiteSpace V (Version No. 3.9.R9 32-bit), which is a visualization Tool Drawing Map of Scientific Knowledge. CiteSpace is a JAVA program visual analysis software, developed by Dr. Chen Chaomei, faculty of Information Science and Technology, Drexel University, USA, to analyze visually network (Chen, 2006) CiteSpace can render huge data as graphics or images intuitively, so that people can understand the multiple attributes of data, and observe the phenomena in a large number of data directly. Visually reflect the focus and provide a basis for research. The research dominant, development trends and sudden changes in the dynamic development network structure of a discipline knowledge field can be searched and displayed when the research objects are analyzed by the map of scientific knowledge (Figure 3 ).

\section{Result and Discussion}

Country Distribution of Beetroot Juice

In the CiteSpace, selected the parameter "country" in analysis software and run it. Chart 2 which is the geographic network map of beetroot juice research field is obtained. Each node is a country; the circle is larger, the publishing articles are more; the thickness of the lines is proportional to the degree of their close relation; the darker the color, the longer the time; the outermost circle represents the centrality of literature. Centrality is a measure of the link in the knowledge map network; the wider of the circle, the greater its centrality, and it is the hub of linking other literature.

Further analysis show that 170 literature are collected from 13 countries. From the number of publications, USA ranks the top (85), followed by UK (18), Andorra (12), Australia (11), Canada (7), Spain (5), Brazil (4), in the middle position. Sweden (3) and Belgium (3) are the last. From the analysis of centrality, the subsequence is USA (1.26), Australia (0.51), UK (0.36), Brazil (0.28), Andorra (0.15), Canada (0.02), Spain (0.01), Sweden (0.00), Belgium $(0.00)$.

Overall, these results indicate that 


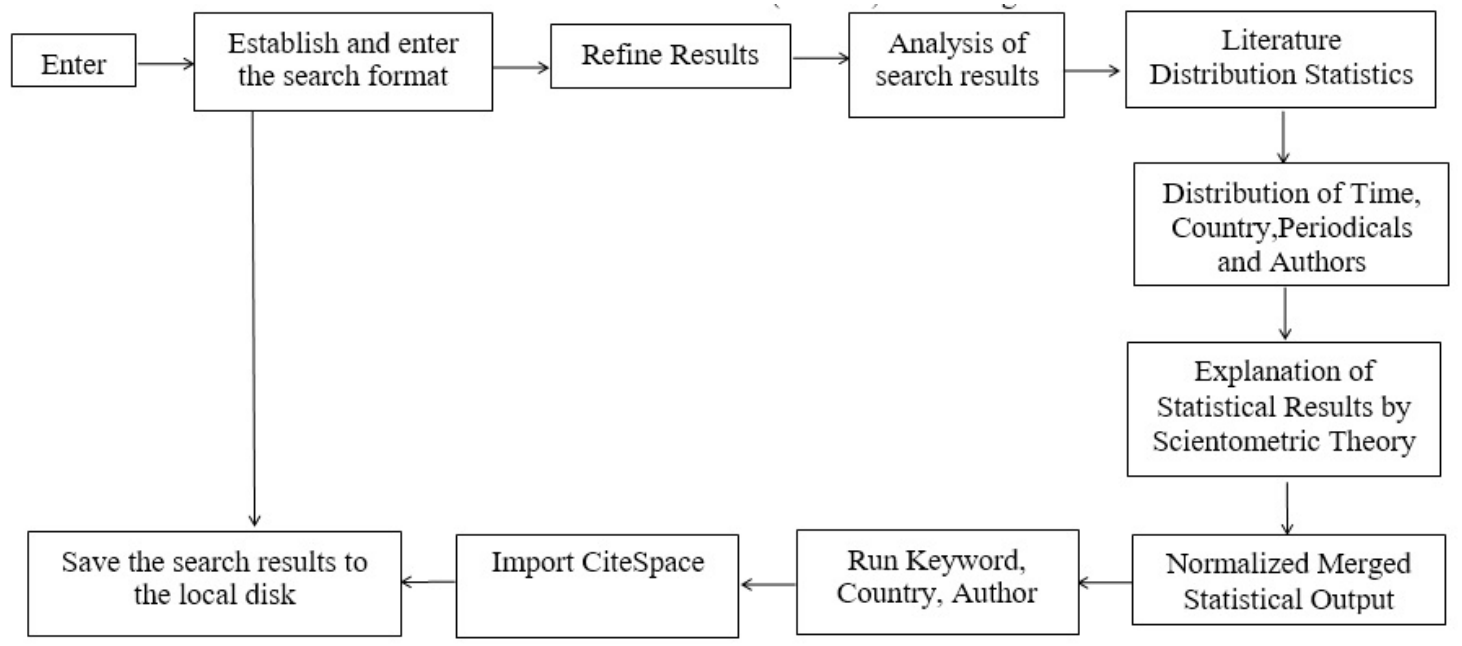

Fig3. Process flow

almost half of studies come from USA, and the centrality is the highest. Although some countries have fewer number of studies, the centrality is higher. Higher centrality means more importance in studies.

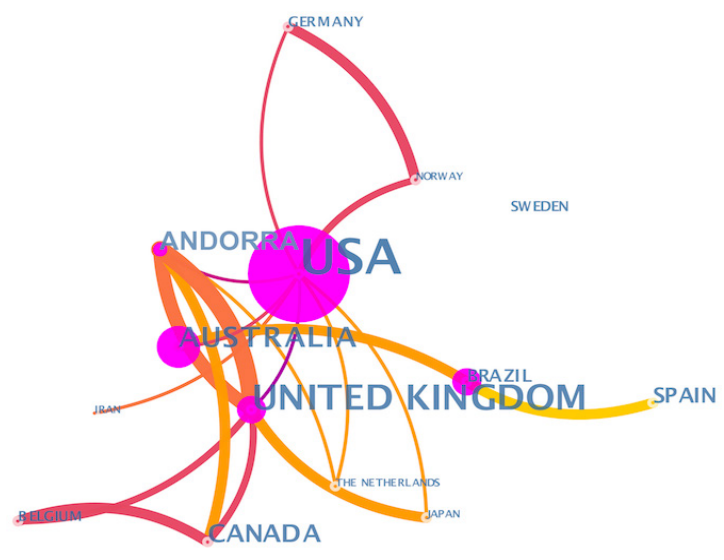

Chart 2. The country map of beetroot juice research

Institution Distribution of Beetroot Juice

The dominant institution are highlighted in Chart 3. The results of the correlational analysis are presented that colleges and universities are dominant in the research of beetroot juice during exercise efforts. Analysis from the frequency of publication, University of Illinois (USA), Universitat d'Andorra (Andorra), Duke University (USA), University of Exeter Medical Department (UK), Queen's University (Canada) are the highest.

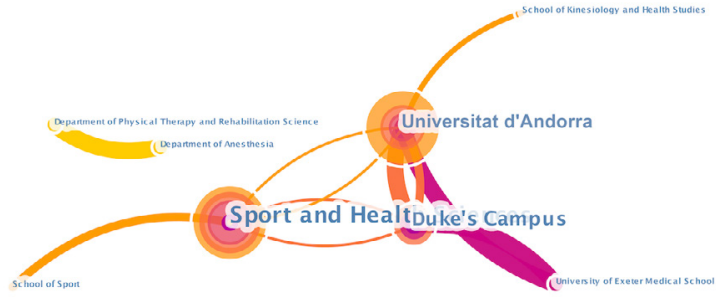

Chart 3. The instituation map of beetroot juice research

Together these results provide important insights into that there are 3 dominant groups: the first one takes School of Sport and Health Sciences of Amercian University of Illinois as the core, followed by Universitat d'Andorra group, and the last one is Duke University group. From the link we can identify that the cooperation of them are deeply and for a long time. What is striking about the figures in this chart is University of Exeter Medical Department (UK) and Duke's Campus were the earliest research institute emerged, and the cooperation of them were more closely. However, they are overtaken by University of Illinois (UI) gradually. UI has the largest sport and health department in the USA and the most advanced experimental equipment for researching, though was established from 1982. The Time Zone of beetroot juice 


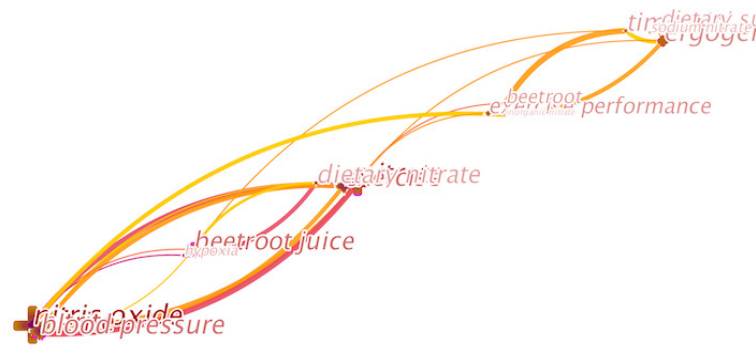

Chart 4. The time zone visualizing map of the theme evolution path

In the CiteSpace, the "Term Source" is selected "Title", "Abstract" and "Author Keywords DE" Threshold remains default, i.e. the first 40 high frequency or high cited nodes; "Node Types" was selected "keywords", and the visual mapping result was "Time Zone", which showed the theme evolution path of beetroot juice research. It is necessary to merge keywords with the same or overlapping meanings in the research after importing the data into the analysis software, for the same keyword may have different expressions in researches. Therefore, integrating keywords and calculating the frequency are great significant to scientific processing of the data. For instance, "Fitness" and "Conditioning" are basically same concept, and "Injury", "Injuries" etc. are merged according to the purpose of the studies. Finally, table 2 is obtained, which is the part of high frequency and centrality keyword lists.

Moreover, in the visualization map, each node represents a keyword of beetroot juice researched in exercise efforts. The size of fonts and nodes are proportional to the frequency of keywords in this research field. The connection between nodes indicates the relationship of two keywords. The thickness represent the degree of relativity of them. So nodes with high citation frequency and centrality have constituted the current prominent research areas between 2010 and 2019. In addition, What stands out in the chart is that beetroot juice as the ergogenic aid in exercise is the main research trend nowadays. Author Cooperative Network Structure

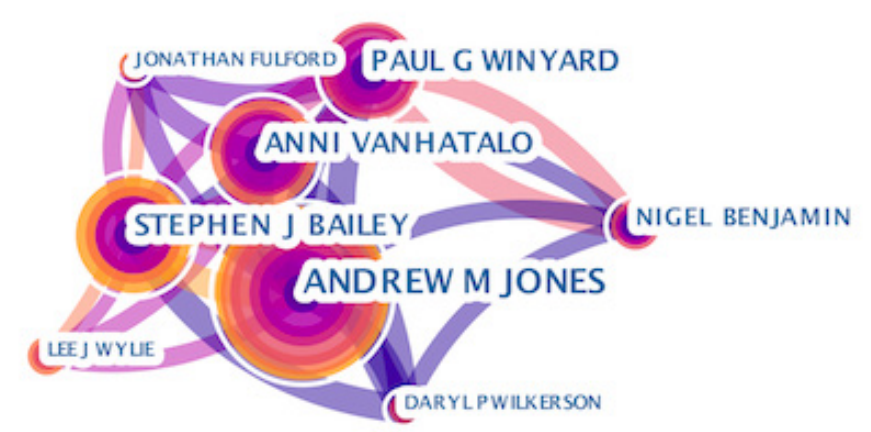

Chart 5. Authors cooperation network view

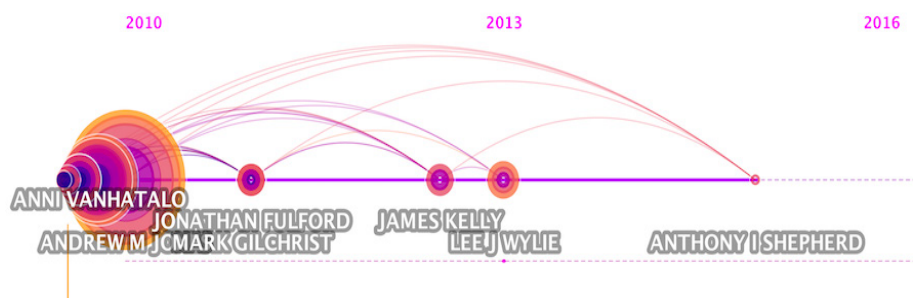

Chart 6. Authors cooperation timeline view

As can be seen from Chart 5 and Chart 6 , there exists the largest group of authors' cooperation network and three sub-groups, which constituted a dominant position. Conclude from Table 3, they are Anni Vanhatalo $(\mathrm{C}=0.12)$, Andrew M. Jones $(\mathrm{C}=0.23)$ and Jonathan fulford $(\mathrm{C}=0.15)$, Jcmark Gilchrist $(\mathrm{C}=0.11)$ and James P. Kelly $(\mathrm{C}=0.07)$ and Lee J. Wylie $(\mathrm{C}=0.05)$ respectively. The first group connection lines, the color difference are more obvious, indicating stable cooperative relationship.

Revealing the prominent research field

In order to explore the prominent research field of beetroot juice in exercise efforts, summarized researches of core group, scholars and emerging cooperation groups. Different research areas are revealed by core scholars of different groups. 
Table 3. Core Scholars and Work Institutions.

\begin{tabular}{ccccc}
\hline & Core Researcher & Frequency & Centrality & Institution \\
\hline 1 & Andrew M. Jones & 37 & 0.23 & University of Exeter Medical Department \\
2 & Anni Vanhatalo & 20 & 0.12 & Sport and Health Sciences \\
3 & Jonathan Fulford & 15 & 0.15 & Peninsula College of Medicine and Dentistry \\
4 & Lee J. Wylie & 13 & 0.05 & Sport and health science \\
5 & James P. Kelly & 9 & 0.07 & health concern in the United States and worldwide \\
6 & Jc Mark Gilchrist & 3 & 0.05 & School of Sport and Health Sciences \\
\hline
\end{tabular}

Established effects of dietary $\mathrm{NO}_{3}^{-}$ supplementation

a. Blood pressure

Over the past decade, most research in blood pressure (BP) has emphasized the use of fruit and vegetables, which can benefit to cardiovascular health (Appel et al., 1997; Gilchrist, Winyard, \& Benjamin, 2010). (Bailey et al., 2014; Vanhatalo et al., 2010) argue that $\mathrm{BR}$ as $\mathrm{NO}_{3}^{-}$supplementation can obviously decrease BP in health adults after acute and chronic supplementation . Especially, (Webb, A. et al., 2008) found that systolic and diastolic can be lowered after ingest the acute beetroot juice, by $\sim 10.4 \mathrm{mmHg}$ and $\sim 8 \mathrm{mmHg}$ respectively, and this change will be recovered to standard value by $24 \mathrm{~h}$. Normally, rising NO bioavailability improve muscle smoothly through synthesis of cyclic guanosine monophosphate (CGMP) (Murad \& Monophosphate, 1986), which is the reason cause the BP reduction. Therefore, increasing dietary $\mathrm{NO}_{3}^{-}$supplementation can have a effect on theraputic and prophylactic inervention for lowering the risk of high $\mathrm{BP}$. Recently, there has been renewed interest in different kinds of BR, for concentrated BR ( $\sim 0.058 \mathrm{mmol} / \mathrm{ml} \mathrm{NO}_{3}^{-}$) emerges, which provide condition to investigate the dose-response relationship, by comparison there was only non-concentrated $\mathrm{BR}\left(0.01 \mathrm{mmol} / \mathrm{ml}^{\mathrm{NO}_{3}}{ }^{-}\right)$ previously. This issue is the foundamental of $\mathrm{BR}$ applied in exercise efforts.

b. $\mathrm{O}_{2}$ cost during submaximal exercise

In the beginning, researches showed that if the submaximal exercise performed under gas change threshold, $\mathrm{VO}_{2}$ will stay stably after 2 3min, which means metabolic cost during exercise, and it is considered unaffected by physical condition (e.g. age, health etc.) (Jones \& Poole, 2005). However, near future, (Larsen et al., 2007) first indicate that the beetroot as the $\mathrm{NO}_{3}^{-}$supplementation has the significant effect on the physiological response during submaximal exercise. The research was taken by nine trained subjects. The result show that $\mathrm{NO}_{3}$ - supplementation increased plasma by $82 \%$, While reduce $\mathrm{VO}_{2}$ by $5 \%$ in the submaximal exercise, which $\mathrm{VO}_{2 \max }$ is maintained at $40 \% \sim 85 \%$. In order to promote exercise efficiency, lowering $\mathrm{VO}_{2}$ after $\mathrm{NO}_{3}{ }^{-}$supplementation ingestion has a positive effect during submaximal exercises (Vanhatalo et al., 2010)Further, with eight recreationally active males and females, cycling test which maintains $\mathrm{VO}_{2 \text { peak }} 47 \pm 8 \mathrm{ml} . \mathrm{kg}^{-1} \cdot \mathrm{min}^{-1}$ following ingestion acute $\mathrm{BR}$ supplementation $(2.5 \mathrm{~h}$ prior to exercise), $5 \mathrm{~d}$ and $15 \mathrm{~d}$ of $\mathrm{NO}_{3}^{-}$rich $\mathrm{BR}$ supplementation $\left(\sim 5.2 \mathrm{mmol} \mathrm{NO}_{3}^{-} \cdot\right.$ day $\left.^{-1}\right)$. The evidence

c. Exercise performance

Since 2010, plasma $\left[\mathrm{NO}_{2}^{-}\right]$has identified that it has an interaction effect in exercise tolerance (Drei冈igacker et al. 2010), $\mathrm{BR}$ as the dietary $\mathrm{NO}_{3}^{-}$supplementation can be an ergogenic aid to benefit the performance during exercise efforts were paid more and more attention by researchers. (Jones, A., Wilkerson, D., DiMenna, J., \& Poole, D., 2008) emphasize that rich BR supplementation with $\sim 5.6 \mathrm{mmol} \mathrm{NO}_{3}^{-}$daily for six days has been shown to improve plasma $\left[\mathrm{NO}_{2}^{-}\right]$, thereby, increase exercise time until limit of tolerance. In addition, (Vanhatalo et al., 2010) highlight that there is a significant rise in peak power output after 8 recreationally active males and females ingest actue $\mathrm{BR}\left(\sim 5.2 \mathrm{mmol} \mathrm{NO}_{3}^{-} \cdot\right.$ day $\left.^{-1}\right) 15$ 
days, but 2.5 days and 5 days are ineffective. And consistent with verification of Vanhatalo, acute $\mathrm{NO}_{3}$ - supplementation can benefit performance in elite cyclists during 4- and $16,1 \mathrm{~km}$ time trial (Larsen et al., 2007) and $10 \mathrm{~km}$ time trial (Cermak et al., 2012). However it is ineffective in elite runners who ingest acute $(2.5 \mathrm{~h}$ prior to $5 \mathrm{~km}$ time trial) of $\mathrm{NO}_{3}$ supplementation (9.9 $\mathrm{mmol} \mathrm{NO}_{3}^{-}$) (Sandbakk et al., 2015), and $2.5 \mathrm{~h}$ prior to $1500 \mathrm{~m}$ time trial with $19.5 \mathrm{mmol}$ $\mathrm{NO}_{3}^{-}$(Breese et al., 2013). Reasons for this is unclear until now, but may be due to the stronger skeletal muscle Blood capillary (Jones, 2008), which can contribute to the decrease of hypoxia condition, a necessary environment for $\mathrm{NO}_{2}^{-}$- NO pathway (Castello, David, Mcclure, Crook, \& Poyton, 2006). Eventually, the concentration is transferred to type II muscle (Wylie et al., 2013) which is recruited lower proportion during endurance exercise (Tesch \& Karlsson, 1985). Therefore, the efficacy of $\mathrm{NO}_{3}$ supplementation to improve the performance of athletes should consider many factors, such as participants, protocols and dose, etc. This is one of the prominent field in beetroot juice research.

B.The Optimal Strategy for Dietary $\mathrm{NO}_{3}$ Supplementation

From the previous literature, it is clear that the acute and chronic $\mathrm{NO}_{3}^{-}$supplementation can lower the $\mathrm{O}_{2}$ cost during submaximal exercise, thus can improve performance in exercise, especially in recreational athletes. Consequently, $\mathrm{BR}$ as a kind of $\mathrm{NO}_{3}^{-}$ supplementation has a significant increase in researches, which are evaluated the effect on different exercise parameters. So, optimal strategy is the focus of research, which include different dose, protocols and participants, etc. From the analysis of author cooperation network, the dose has an exponential rise in the research.

Wylie et al., (2013) tested 14 male participants who were team-sport players ingested nitrate-rich BR, and adopted Yo-Yo IR1. There was a significant improvement $400 \%$ greater resting plasma $\left[\mathrm{NO}_{2}^{-}\right]$concentration compared to the placebo group. Another previous study from (Thompson, Vanhatalo, et al., 2015) highlighted BR had the effect on performance during Yo-Yo IR1. In this study, a double-blind, randomized, crossover experiment involved 36 male team-sport players who ingested rich $\mathrm{BR}\left(6.4 \mathrm{mmol} \mathrm{NO}_{3}{ }^{-}\right)$ and $\mathrm{NO}_{3}{ }^{-}$depleted $\mathrm{BR}\left(0.04 \mathrm{mmol} \mathrm{NO}_{3}^{-}\right)$, and completed Yo-Yo IR1. However, there is still a lot need to prove about effect in Yo-Yo IR1, such as different level trained athletes, gender, even dose of BR.

Several studies focus on chronic strategy and have evaluated the physiological response to exercise, but an acute $\mathrm{NO}_{3}$ - supplementation has been provided prior to the evaluation to ensure the plasma $\left[\mathrm{NO}_{2}^{-}\right.$] was obviously rose (Vanhatalo et al., 2010). Thus, these studies did not only focus on the chronic supplementation, but combined the effect of chronic and acute ingestion, which is the important practice of optimal strategy for BR.

C. Potential effects of dietary $\mathrm{NO}_{3}^{-}$ supplementation on intermittent exercise performance

Historically, research investigating the factors associated with BR has focused on endurance exercise, especially in moderatelytrained athletes can be improved performance (Muggeridge, Howe, Spendiff, Pedlar, James, \& Easton, 2013) However, there is a exponential increase in high-intensity intermittent exercise, evolved some most popular competitions such as soccer, basketball, etc. This kind of exercise usually intersperse with periods of recovery. Therefore, a correlation exists from a low to high metabolic rate repeatedly, which evolve anaerobic and aerobic energy pathways (Kelly et al., 2014; Kelly, Wilkerson, Wylie, \& Jones, 2013). This prominent area include muscle fiber type recruited, Exercise-inducing fatigue and Yo-Yo intermittent recovery test.

a. Muscle fibers type recruited

Research into muscle fibers recruited during exercise effort has a long history. It is well know type II muscle fibers recruited in power generation. (Webb, A. et al., 2008) And some studies also find that compare to type I muscle fibers, type II fibers deplete phosphocreatine and glycogen greater during high-intensity exercise (Volek, J. et al., 1997). So, with the deepening of investigations, researchers are beginning to pay attention to BR benefit highintensity intermittent exercise, for type II fibers recruited a lot during such exercise. (Wylie 
Table 4. Yo-Yo IR1 test protocol After each 23 20-m shuttle run bout, players perform 10 seconds of active recovery jogging round a cone set $5 \mathrm{~m}$ apart from the starting line.

\begin{tabular}{lllll}
\hline & Speed $\left(\mathrm{km} \cdot \mathrm{h}^{-1}\right)$ & Shuttle & Split Distance $(\mathrm{m})$ & Accumulated Distance $(\mathrm{m})$ \\
\hline 1 & 10 & 1 & 40 & 40 \\
2 & 12 & 1 & 40 & 80 \\
3 & 13 & 2 & 80 & 160 \\
4 & 13.5 & 3 & 120 & 280 \\
5 & 14 & 4 & 160 & 440 \\
6 & 14.5 & 8 & 320 & 760 \\
7 & 15 & 8 & 320 & 1080 \\
8 & 15.5 & 8 & 320 & 1400 \\
9 & 16 & 8 & 320 & 1720 \\
10 & 16.5 & 8 & 320 & 2040 \\
11 & 17 & 8 & 320 & 2360 \\
12 & 17.5 & 8 & 320 & 2680 \\
13 & 18 & 8 & 320 & 3000 \\
\hline
\end{tabular}

et al., 2016) indicate that NO has the effect to increase blood flow caused by $\mathrm{NO}$ is definite to type II muscle fibers. In addition, (Kelly, Wilkerson, et al., 2013) argue that the calcium from the sarcoplasmic reticulum can be enhaced to release and resorb by BR ingestion in type II muscle fibers which can translate to a rise ability for force generation. This could be a significant advantage for exercise efforts recruiting the type II muscle fibers during high-intensity intermittent exercise. Hence, increasing number of studies have appeared to address this issue, and become one of the prominent research area in BR during a particular exercise effort.

b. Yo-Yo intermittent recovery test (IR1)

Yo-Yo IR1 is a famous method to simulate the high-intensity intermittent bouts, especially in team sport (Castagna, Impellizzeri, Rampinini, Ottavio, \& Manzi, 2008) (Table 4).

\section{Conclusion}

The United State and European countries occupied a dominant position in the carriers of research network. Current prominent research areas were effects of dietary $\mathrm{NO}_{3}$ supplementation, including blood pressure, $\mathrm{O}_{2}$ cost of submaximal exercise, and exercise performance. The optimal strategy for dietary $\mathrm{NO}_{3}{ }^{-}$supplementation and potential effects of dietary $\mathrm{NO}_{3}{ }^{-}$supplementation on intermittent exercise performance involve muscle fibre type recruited and the Yo-Yo intermittent recovery test.

\section{References}

Appel, L. J., Moore, T. J., Barzanek, E. O., Vollmer, W. M., Vetkey, L. P. S., Sacks, F. M., ... Karanja, N. J., 1997. A Clinical Trial Of The Effects Of Dietary Patterns On Blood Pressure. Journal of Medicine, 336(16), 1117-1124.

Aucouturier, J., Boissiere, J., Pawlak-chaouch, M., Cuvelier, G., \& Gamelin, F.-X., 2015. Effect of dietary nitrate supplementation on tolerance to supramaximal intensity intermittent exercise. Nitric Oxide Journal, 49, 16-25. https://doi.org/10.1016/j.niox.2015.05.004

Bailey, S. J., Fulford, J., Vanhatalo, A., Winyard, P. G., Jamie, R., Dimenna, F. J., ... Jones, A. M., 2014. Dietary nitrate supplementation enhances muscle contractile efficiency during knee-extensor exercise in humans and heavy bilateral knee extension. Journal of Applied Physiology, 109(1), 135-148. https:// doi.org/10.1152/japplphysiol.00046.2010

Breese, B. C., Mcnarry, M. A., Marwood, S., Blackwell, J. R., Bailey, S. J., \& Jones, A. M., 2013. Beetroot juice supplementation speeds $\mathrm{O} 2$ uptake kinetics and improves exercise tolerance during severe-intensity exercise initiated from an elevated metabolic rate. Amercian Journal of Physiology Regulatory, Integrative and Comparative Physiology, 305(12). https://doi.org/10.1152/ ajpregu.00295.2013

Castagna, C., Impellizzeri, F. M., Rampinini, E., Ottavio, S. D., \& Manzi, V., 2008. The Yo Yo intermittent recovery test in basketball players. 202-208. https://doi.org/10.1016/j. jsams.2007.02.013

Castello, P. R., David, P. S., Mcclure, T., Crook, 
Z., \& Poyton, R. O., 2006. Mitochondrial cytochrome oxidase produces nitric oxide under hypoxic conditions: Implications for oxygen sensing and hypoxic signaling in eukaryotes. (April), 277-287. https://doi. org/10.1016/j.cmet.2006.02.011

Cermak, N. M., Res, P., Stinkens, R., Lundberg, J. O., Gibala, M. J., \& Loon, L. J. C. Van., 2012. No Improvement in Endurance Performance After a Single Dose of Beetroot Juice. International Journal of Sport Nutrition and Exercise Metabolism, 22, 470-478.

Chen, C., M., 2017. A set of demonstrations on how to use CiteSpace.

Chen, C., 2006. CiteSpace II : Detecting and Visualizing Emerging Trends. Journal of the Association for Information Science and Technology, 57(3), 359-377. https://doi. org/10.1002/asi

Domínguez, R., Maté-muñoz, J. L., Cuenca, E., García-fernández, P., Mata-ordoñez, F., Lozano-estevan, M. C., ... Garnachocastaño, M. V., 2018. Effects of beetroot juice supplementation on intermittent high-intensity exercise efforts. Journal of the International Society of Sports Nutrition (2018), 15(2), 1-12. https://doi.org/10.1186/ s12970-017-0204-9

Gilchrist, M., Winyard, P. G., \& Benjamin, N., 2010. Nitric Oxide Dietary nitrate - Good or bad ? Nitric Oxide, 22(2), 104-109. https://doi. org/10.1016/j.niox.2009.10.005

Jones, A., M., Wilkerson, D., P., DiMenna, F., J., F., \& Poole, D., C., 2008. Muscle metabolic responses to exercise above and below the "critical power" assessed using 31P-MRS. Amercian Journal of Physiology Regulatory, Integrative and Comparative Physiology, 294(2), R5.

Jones, A. M., \& Poole, D. C., 2005. Oxygen Uptake Dynamics: From Muscle to Mouth - An Introduction to the Symposium. Medicine and Science in Sports and Exercise, 37(9), 1542-1550. https://doi.org/10.1249/01. mss.0000177466.01232.7e

Kelly, J., Vanhatalo, A., Bailey, S. J., Wylie, L. J., Tucker, C., List, S., ... List, S., 2014. Dietary nitrate supplementation: effects on plasma nitrite and pulmonary $\mathrm{O} 2$ uptake dynamics during exercise in hypoxia and normoxia. Am J Physiol Regul Integr Comp Physiol, 307, 920-930. https://doi.org/10.1152/ ajpregu.00068.2014

Kelly, J., Vanhatalo, A., Wilkerson, D. P., Wylie, L. E. E. J., \& Jones, A. M., 2013. Effects of Nitrate on the Power-Duration Relationship for
Severe-Intensity Exercise. Medicine \& Science in Sports \& Exercise, (3), 1798-1806. https:// doi.org/10.1249/MSS.0b013e31828e885c

Kelly, J., Wilkerson, D. P., Wylie, L. J., \& Jones, A. M., 2013. Effects of Nitrate on the Power-Duration Relationship for Severe-Intensity Exercise. Medicine \& Science in Sports \& Exercise, Publish Ahead of Prin, (February). https:// doi.org/10.1249/MSS.0b013e31828e885c

Larsen, F. J., Weitzberg, E., Lundberg, J. O., \& Ekblom, B., 2007. Effects of dietary nitrate on oxygen cost during exercise. Acta Physiol, 191, 59-66. https://doi.org/10.1111/j.17481716.2007.01713.x

Lundberg, J.O., \& Govoni, M., 2004. Inorganic Nitrate Is A Possible Source For Systemic Generation Of Nitric Oxide. Free Radical Biology \& Medicine, 37(3), 395-400. https://doi. org/10.1016/j.freeradbiomed.2004.04.027

Muggeridge, D. J., Howe, C. C. F., Spendiff, O., Pedlar, C., James, P. E., \& Easton, C., 2013. A Single Dose of Beetroot Juice Enhances Cycling Performance in Simulated Altitude. Medicine \& Science in Sports \& Exercise, (29), 143-150. https://doi.org/10.1249/ MSS.0b013e3182a1dc51

Muggeridge, D. J., Howe, C. C. F., Spendiff, O., Pedlar, C., James, P. E., Easton, C., \& Less, V., 2013. The Effects of a Single Dose of Concentrated Beetroot Juice on Performance in Trained Flatwater Kayakers. Https://Www. Ncbi.Nlm.Nih.Gov/Pubmed/23580456\#, 23(5), 498-506.

Murad, F., \& Monophosphate, C. G., 1986. Cyclic guanosine monophosphate as a mediator of vasodilation. Find the latest version : Journal Clinic Investigation, 78(1), 1-5.

Pinna, M., Roberto, S., Milia, R., Marongiu, E., Olla, S., Loi, A., ... Crisafulli, A., 2014. Effect of Beetroot Juice Supplementation on Aerobic Response during Swimming. Nutrients, 6(3), 605-615. https://doi.org/10.3390/nu6020605

Richardson, R. S., Leigh, J. S., \& Wagner, P. D., 1995. Myoglobin $\mathrm{O} 2$ desaturation during exercise . Evidence of limited O2 transport. Find the latest version: Desaturation during Exercise. 96(4), 1916-1926.

Tesch, A. P., \& Karlsson, J. A., 1985. Muscle fiber types and size in trained and untrained muscles of elite athletes. Journal of Applied Physiology, 59(1716).

Thompson, K., G., Turnerb, L., Prichardb, J., Doddb, F., Kennedyb, D., O., Haskellb, C., ... Jones, A., M., 2014. Influence of dietary nitrate supplementation on physiological and cognitive responses to incremental cycle 
exercise. Respirator Physiology Neurobiology, 193(3), 11-20.

Thompson, C., Vanhatalo, A., Jell, H., Fulford, J., Carter, J., Nyman, L., ... Jones, A. M., 2015. Dietary Nitrate Supplementation Improves Sprint And High-Intensity Intermittent Running Performance. European Journal Applied Physiology, 115(9), 1825-1834.

Thompson, C., Wylie, L. J., Fulford, J., \& Kelly, J., 2015. Dietary nitrate improves sprint performance and cognitive function during prolonged intermittent exercise. European Journal of Applied Physiology, 1825-1834. https://doi.org/10.1007/s00421-015-3166-0

Todd, D. H., Grabowski, P. S., Helfrich, M., Benjamin, N., \& Ralston, S. H., 1994. O3 Nitric oxide production in cultures of human osteoblastlike cells. Bone, 15(4), 449-450.

Vanhatalo, A., Bailey, S. J., Blackwell, J. R., Dimenna, F. J., Pavey, T. G., Wilkerson, D. P., ... Jones, A. M., 2010. Acute and chronic effects of dietary nitrate supplementation on blood pressure and the physiological responses to moderate-intensity and incremental exercise. Am J Physiol Regul Integr Comp Physiol 299:, 299(4), 1121-1131. https://doi.org/10.1152/ ajpregu.00206.2010.

Volek, J., S., Kraemer, W., J., Bush., J., A., Boetes, M., Incledon, T., Clark, K., L., \& Lynch, J.,
M., 1997. Creatine supplementation enhance muscular performance during high-intensity resistance exercise. Journal of the American Dietetic Association, 97(7), 765.

Webb, A., J., Patel, N., Loukogeorgakis, S., Okorie, M., Aboud, Z., Misra, S., ... Ahluwalia, A., 2008. Acute blood pressure lowering, vasoprotective, and antiplatelet properties of dietary nitrate via bioconversion to nitrite. Hypertension, 51(3), 784-790.

Wylie, L. J., Bailey, S. J., Kelly, J., Blackwell, J. R., Vanhatalo, A., \& Jones, A. M., 2016. Influence of beetroot juice supplementation on intermittent exercise performance. European Journal of Applied Physiology, 116(2), 415425. https://doi.org/10.1007/s00421-0153296-4

Wylie, L. J., Mohr, M., Krustrup, P., Jackman, S. R., Vanhatalo, A., \& Jones, A. M., 2013. Dietary nitrate supplementation improves team sport-specific intense intermittent exercise performance. Eur J Appl Physiol (2013), 113, 1673-1684. https://doi.org/10.1007/s00421013-2589-8 